\title{
Dermal dendritic cell number correlates with serum autoantibody titers in Brazilian pemphigus foliaceus patients
}

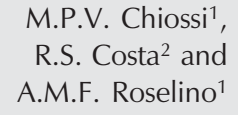

${ }^{1}$ Divisão de Dermatologia, Departamento de Clínica Médica, and ${ }^{2}$ Departamento de Patologia, Faculdade de Medicina de Ribeirão Preto, Universidade de São Paulo, Ribeirão Preto, SP, Brasil

\author{
Correspondence \\ A.M. Roselino \\ Divisão de Dermatologia \\ Departamento de Clínica Médica \\ FMRP, USP \\ Av. Bandeirantes, 3900 \\ 14049-900 Ribeirão Preto, SP \\ Brasil \\ Fax: +55-16-633-6695 \\ E-mail: amfrosel@fmrp.usp.br \\ Research partially supported by \\ $\mathrm{CNPq}$ and FAPESP. \\ Part of these data have been \\ presented at the National Pemphigus \\ Foundation and the American \\ Autoimmune Related Disease \\ Association International Meeting: \\ Pemphigus as a Model of \\ Organ-Specific Humoral Autoimmune \\ Diseases. Bethesda, MD, USA, April \\ 20-21, 2001.
}

Received June 9, 2003

Accepted November 6, 2003

\begin{abstract}
Pemphigus foliaceus (PF) is an autoimmune bullous disease endemic in Brazil. Since serum IL-12 is increased in patients with PF and Langerhans cells (LC) produce IL-12, we titrated serum autoantibodies by indirect immunofluorescence, and quantified epidermal dendritic cells, known as LC, and dermal dendritic cells (DC). Biopsies of blistering lesions were obtained from 22 patients, 13 of whom were submitted to biopsy of both injured and of apparently healthy skin. The control groups consisted of skin from 8 cadavers and from 12 women submitted to breast plastic surgery. LC and DC were identified with anti-CD1a antibody and quantified by morphometric analysis. LC number in the lesion and in apparently healthy skin from PF patients was similar to that of both control groups. DC number in the injured skin (median $=0.94 \mathrm{DC} / \mathrm{mm}$ basement membrane) was higher than that of the cadaver group (median $=0.13 \mathrm{DC} / \mathrm{mm}$ basement membrane). In the 13 patients with biopsies of both injured and apparently healthy skin, LC and DC were present in larger numbers in the lesion. There was a direct correlation between DC number in the lesion of the $\mathrm{PF}$ group and serum autoantibody titers. This correlation was not observed for LC number. The increased number of DC in the lesion, as well as its direct correlation with serum autoantibody titers suggest the participation of $\mathrm{DC}$ in the pathogenesis of $\mathrm{PF}$. The relationship between increased DC number and IL-12 in PF needs to be clarified.
\end{abstract}

Key words

- Anti-CD1a

- Dendritic cells

- Fogo selvagem

- Langerhans cells

- Pemphigus foliaceus
Pemphigus foliaceus (PF) is an autoimmune bullous disease characterized by acantholysis in the lower layer of the stratum corneum of the epidermis, deposition of immunoglobulin $\mathrm{G}(\mathrm{IgG})$ in the intercellular space of the epidermis, and circulating $\operatorname{IgG}$ against the intercellular substance, as demonstrated by indirect immunofluorescence (IIF). The autoantibodies are directed against the glycoprotein desmoglein 1 (Dg1) of des- mosomes $(1,2)$. PF is endemic in Brazil and we recently demonstrated that the northeastern region of the State of São Paulo is a remaining focus of $\mathrm{PF}(3,4)$.

Although there is great interest in the study of humoral immunity, few reports describe the involvement of cell immunity associated with PF. Among the cellular elements of the skin, dendritic cells (DC) and the subgroup of Langerhans cells (LC) are of 
special interest. LC are epidermal dendritic cells with antigen-presenting and $\mathrm{T}$ lymphocyte-activating abilities $(5,6)$. The LC population is reduced in the epidermis of $\mathrm{PF}$ patients and the number of dermal DC is higher in the lesional skin than in the perilesional skin (7).

DC produce cytokines which can induce $\mathrm{T}$ cell differentiation, and among them IL-12 has a primordial role by inducing the Th1 phenotype $(8,9)$. In the serum of PF patients, IL-4, IL-5 and INF- $\gamma$ are decreased, and IL10 and IL-12 are increased compared to controls (10). The production of IL-4, IL-5 and IL- 6 but not of IFN- $\gamma$ by T lymphocytes that specifically proliferate in response to Dg1 has been detected in patients with PF, suggesting that a Th2 pattern participates in its pathogenesis (11).

Since serum IL-12 is increased in PF (10) and DC, which produce IL-12, have an antigen-presenting function, the purpose of the present study was to quantify LC and DC in the skin of patients with PF and to attempt to correlate their numbers with circulating serum autoantibody titers.

Twenty-two patients with a diagnosis of PF, with active disease (positive Nikolsky signal), attended at the University Hospital of the Faculty of Medicine of Ribeirão Preto, University of São Paulo, were included in the study after giving informed written consent to participate. The protocol was approved by the Hospital Ethics Committee. Patients taking anticoagulant drugs and pregnant women were excluded. Thirteen presented the localized clinical form and 9 the generalized form of PF. Eleven patients were under treatment with prednisone (4 of whom were taking it in combination with chloroquine). Biopsies of both blistering lesion and of healthy skin in a sun-protected area (trunk or proximal lower limbs) were collected from 13 patients on the same day. The reason for using skin not adjacent to a lesion was to avoid detecting alterations of the disease itself, and the reason to use skin from a sun- protected area was due to the reduction of DC in sunlight-exposed areas (12). Eight skin fragments obtained from the anterior thoracic region of cadavers ( $3 \mathrm{~h}$ after death, on average) and 12 skin fragments obtained from 12 women submitted to breast plastic surgery were used as controls. There was no statistical difference concerning race or age between the PF and control groups.

For IIF, a fragment of human skin obtained from an abdominoplasty was used as substrate. Five-micrometer sections were incubated with patient serum at increasing dilutions for $30 \mathrm{~min}$ at room temperature. After rinsing with PBS, the sections were incubated with fluorescein-conjugated antihuman IgG (anti-human IgG-FITC, Behringwerke AG, Marburg, Germany) at 1:20 dilution for $30 \mathrm{~min}$. After rinsing with PBS, the slides were mounted with buffered glycerin. Sections incubated with normal human serum and anti-human IgG were used as a negative control, and slides incubated only with anti-human IgG were used for the control of nonspecific fluorescence.

For LC and DC identification by immunohistochemistry, the OKT6 monoclonal antibody obtained in mice (anti-CD1a, Immunotech, Westbrook, ME, USA) was applied to $5-\mu \mathrm{m}$ sections previously deparaffinized in xylene and hydrated in alcohol. As a secondary antibody, biotinylated antimouse rabbit IgG (Dako A/S, Glostrup, Denmark) was used for $35 \mathrm{~min}$, followed by the streptavidin-biotin-peroxidase complex (StreptAB Complex/HRP, Dako A/S). The sections were then developed with diaminobenzidine. As a negative control, the primary antibody was excluded.

LC and DC quantification was performed by morphometric analysis, and the number of $\mathrm{LC} / \mathrm{mm}^{2}$ epidermis, $\mathrm{LC} / \mathrm{mm}$ stratum corneum (SC), and LC/mm basement membrane (BM) were obtained. LC and DC were counted using light microscopy, with a $40 \mathrm{X}$ magnification objective.

The epidermal dimensions were obtained 
by morphometric analysis using a videocamera coupled to a light microscope fitted with a 10X magnification objective. The image obtained on the video monitor was connected to an automatic image analyzer (KS300 Kontron Imaging System, Kontron Elektronic, Eching bei München, Germany) and epidermal area, BM and SC extension were measured.

Data concerning IIF and LC and DC quantification were analyzed statistically by the Kruskal-Wallis, Mann-Whitney and Wilcoxon tests, and the correlation between IIF titers and cell quantification was calculated by the Spearman test.

In patients with the localized form of the disease, serum antibody titration by IIF showed lower serum antibody titers (1:512 to $1: 2048$, median 1:1024) compared to patients with the generalized form (1:512 to $1: 16384$, median 1:4096; $\mathrm{P}=0.0083$ ).

There was a statistically significant difference $(\mathrm{P}=0.0492)$ in LC quantification (Figure 1) between the two control groups (cadavers and women submitted to plastic breast surgery) both in number of $\mathrm{LC} / \mathrm{mm}$ $\mathrm{BM}$ and number of $\mathrm{LC} / \mathrm{mm} \mathrm{SC}$, a fact that did not permit their pooling into a single population for data analysis.

In the 13 patients with PF from whom skin biopsies were obtained from both apparently healthy and injured skin, the LC number was significantly higher in the lesioned skin when $\mathrm{LC} / \mathrm{mm}^{2}(\mathrm{P}=0.0134)$ and $\mathrm{LC} / \mathrm{mm} \mathrm{SC}(\mathrm{P}=0.0215)$ were quantified, but not significantly higher when $\mathrm{LC} / \mathrm{mm} \mathrm{BM}$ was measured.

DC numbers differed between the control groups $(P=0.0122)$ in dermal DC quantification (Figure 2). In the PF lesion, DC were present in higher numbers $(0.94 / \mathrm{mm}$ $\mathrm{BM})$ than in cadaver skin $(0.13 / \mathrm{mm} \mathrm{BM} ; \mathrm{P}=$ 0.0329). In the same patient of the PF group, the DC number in the injured skin was higher compared to apparently healthy skin $(\mathrm{P}=$ 0.0049).

There was a correlation between dermal
$\mathrm{DC}$ number/mm $\mathrm{BM}$ in the PF lesion and serum antibody titers by IIF $(r=0.4779, \mathrm{P}<$ $0.05)$, whereas this correlation was not sig-

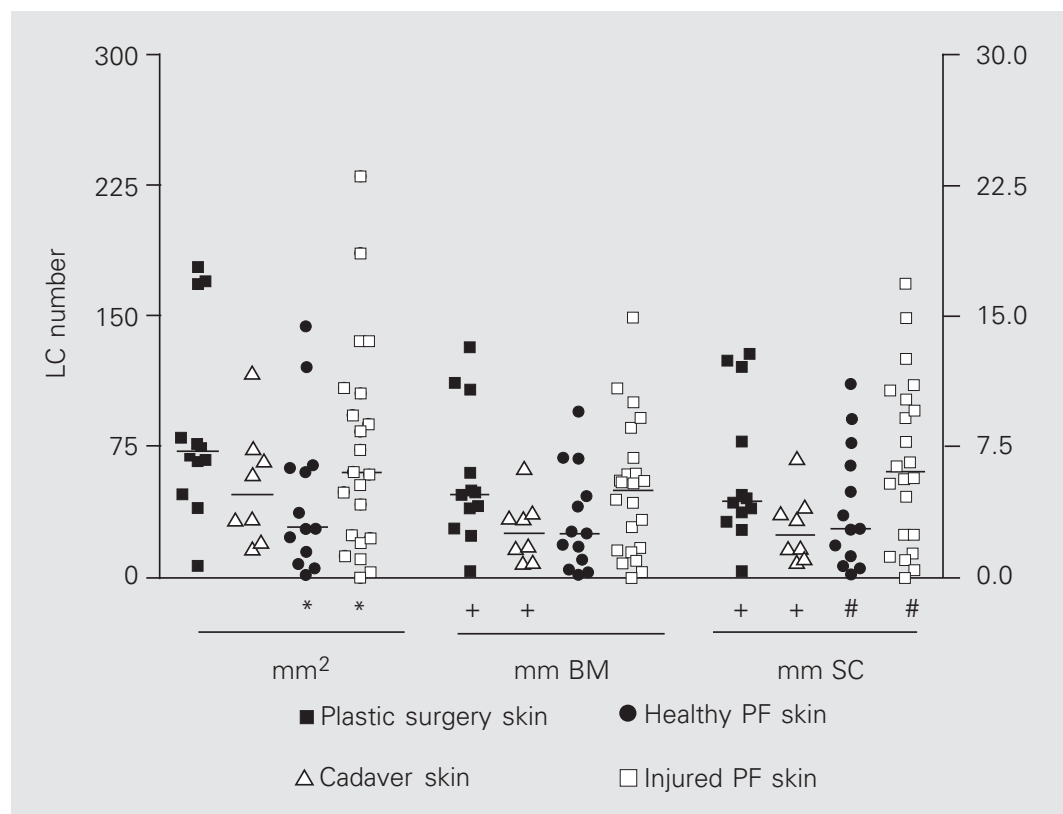

Figure 1. Langerhans cells (LC) in skin biopsies from patients submitted to breast plastic surgery $(N=12)$, from cadavers $(N=8)$, and from healthy $(N=13)$ and injured $(N=22)$ skin of pemphigus foliaceus (PF) patients. LC number/mm basement membrane (BM) and LC number/mm stratum corneum (SC) are indicated on the right $Y$ axis and $L C / \mathrm{mm}^{2}$ of epidermis is on the left $Y$ axis. Horizontal lines are the medians. $+v s+: P=0.0492$ (MannWhitney test); ${ }^{*} v S *$ : $\mathrm{P}=0.0134$, and \# vs \#: $\mathrm{P}=0.0215$ (Wilcoxon test).

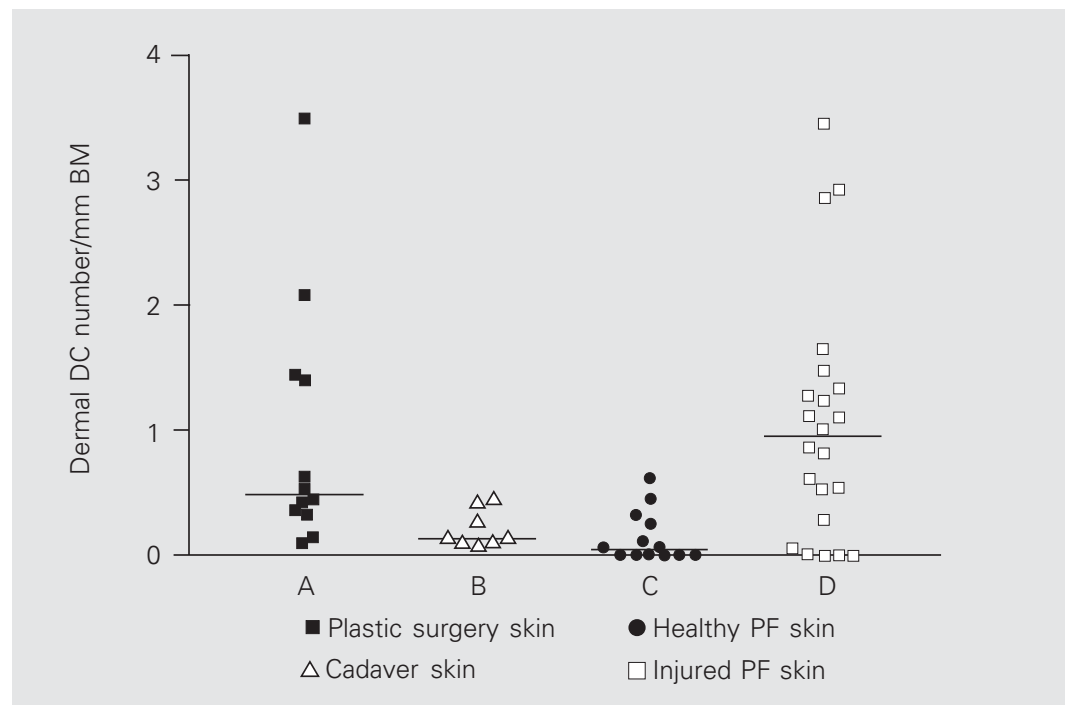

Figure 2. Dermal dendritic cells (DC) in basement membrane (BM). Comparison between skin biopsies from patients submitted to breast plastic surgery $(\mathrm{N}=12)$, from cadavers ( $N$ $=8)$, and from healthy $(N=13)$ and injured $(N=22)$ skin of pemphigus foliaceus (PF) patients. The horizontal lines are the medians. Statistical analysis: $A$ vs B: $P=0.0122$ and $B$ vs D: $\mathrm{P}=0.0329$ (Mann-Whitney test); $\mathrm{C}$ vs $\mathrm{D}: \mathrm{P}=0.0049$ (Wilcoxon test). 
nificant in the apparently healthy skin. This correlation was also not significant for LC number. There are no reports in the literature to permit us to make a comparison of our results concerning correlation between DC number in bullous lesion and serum autoantibody titers in PF.

In the present study we used the antiCD1a monoclonal antibody for LC and DC labeling but we should point out that the values obtained may have been underestimated since DC subpopulations that do not express the CD1a surface antigen were not counted (13), and furthermore the method does not determine whether the cells are functionally active or not (14).

The choice of cadaver skin and skin from women submitted to breast plastic surgery as controls was based on the premise that these skin samples could represent a unique control group, since the DC number detected in these two kinds of skin has been reported to be similar in the literature $(15,16)$. However, we detected higher LC and DC numbers in the skin from patients submitted to plastic surgery, which may have been due to the age difference between the two control groups, since the biopsies were obtained from the same area (anterior thorax). Thus, the cadaver group presented a smaller number of LC, probably because it consisted of older individuals. Another hypothesis could be that in the cadaver group the LC may lose the expression of surface proteins post mortem, although the literature does not show differences between living persons and cadavers $(15,16)$.

The LC number in the PF lesion was similar to that of the control groups. When the blistering lesion was compared to apparently healthy skin in the PF group, the value was higher in injured skin. Only one report by Santi and Sotto (7) is available in the literature, which demonstrates that the LC population was decreased in the lesioned epidermis compared to the perilesional epidermis in PF.

We also point out that in the injured area, due to the bulla, the LC number in some sections may have been underestimated due to inclusion of the bulla in the total area calculated. Thus, when we found a larger number of LC in lesioned skin than in apparently healthy skin of $\mathrm{PF}$ patients, this should be taken into consideration.

The DC number in the blistering lesion of the PF group was increased compared to the cadaver group and was also increased compared to apparently healthy skin. This finding agrees with the results reported by Santi and Sotto (7) in a study comparing lesioned skin with perilesional skin.

When we divided the patients into groups with and without treatment, a higher DC number persisted in the dermis of both of them compared to the cadaver group (data not shown).

Considering the patient group as a whole and overlooking its heterogeneity, we may state that $\mathrm{LC}$ number is unchanged in $\mathrm{PF}$ lesions, whereas the number of dermal DC is significantly increased compared to control groups. In the PF group, both the LC and DC were present in higher numbers in the lesion compared to apparently healthy skin. The significant correlation between serum antibody titers and the number of dermal DC in the PF lesion suggests that DC may play a role in the pathogenesis of PF. The relationship between increased DC number and IL12 in PF needs to be clarified.

\section{References}

1. Warren SJ, Lin MS, Giudice GJ, Hoffmann RG, Hans-Filho G, Aoki V, Rivitti EA, Santos V \& Diaz LA (2000). The prevalence of antibodies against desmoglein 1 in endemic pemphigus foliaceus in Brazil. Cooperative Group on Fogo Selvagem Research. New England
Journal of Medicine, 343: 23-30

2. Diaz LA, Sampaio SA, Rivitti EA et al. (1989). Endemic pemphigus foliaceus (Fogo Selvagem). II. Current and historic epidemiologic studies. Journal of Investigative Dermatology, 92: 4-12. 
3. Chiossi MPV \& Roselino AMF (2001). Endemic pemphigus foliaceus (Fogo Selvagem): a series from the northeastern region of the State of São Paulo, Brazil, 1973-1998. Revista do Instituto de Medicina Tropical de São Paulo, 43: 59-62.

4. Roselino AMF \& Almeida AM (1995). Surto de pênfigo foliáceo endêmico em instituição para menores em Batatais, Estado de São Paulo, Brasil, 1988-1990. Medicina, 28: 44-50.

5. Hogan AD \& Burks AW (1995). Epidermal Langerhans' cells and their function in the skin immune system. Annals of Allergy, Asthma, and Immunology, 75: 5-10.

6. Lappin MB, Kimber I \& Norval M (1996). The role of dendritic cells in cutaneous immunity. Archives of Dermatological Research, 288: 109-121.

7. Santi CG \& Sotto MN (2001). Immunopathologic characterization of the tissue response in endemic pemphigus foliaceus (Fogo Selvagem). Journal of the American Academy of Dermatology, 44: 446450.

8. Romagnani S (1992). Induction of Th1 and Th2 responses: a key role for the "natural" immune response? Immunology Today, 13: 379381.

9. Macatonia SE, Hosken NA, Litton M, Vieira P, Chyi-Song $H$, Culpepper JA, Wysocka M, Trinchieri G, Murphy KM \& O'Garra A (1995). Dendritic cells produce IL-12 and direct the development of Th1 cells from naive CD4+ T cells. Journal of Immunology, 154:
5071-5079.

10. Zeoti DM, Figueiredo JFC, Chiossi MPV \& Roselino AMF (2000). Serum cytokines in patients with Brazilian pemphigus foliaceus (Fogo Selvagem). Brazilian Journal of Medical and Biological Research, 33: 1065-1068.

11. Lin M-S, Fu C-L, Aoki V et al. (2000). Desmoglein-1-specific T lymphocytes from patients with endemic pemphigus foliaceus (Fogo Selvagem). Journal of Clinical Investigation, 105: 207-213.

12. Thiers BH, Maize JC, Spicer SS \& Cantor AB (1984). The effect of aging and chronic sun exposure on human Langerhans cell populations. Journal of Investigative Dermatology, 82: 223-226.

13. Nestle FO \& Nickoloff BJ (1995). Dermal dendritic cells are important members of the skin immune system. Advances in Experimental Medicine and Biology, 378: 111-116.

14. Drijkoningen M, Wolf-Peeter C, Oord Van Den JJ, Degreef H \& Desmet $V$ (1986). Expression of HLA-DR and T6 antigens on keratinocytes and dendritic cells. Archives of Pathology and Laboratory Medicine, 110: 321-325.

15. Berman B, Chen VL, France DS, Dotz WI \& Petroni G (1983). Anatomical mapping of epidermal Langerhans cell densities in adults. British Journal of Dermatology, 109: 553-558.

16. Chen H, Yuan J, Wang $Y$ \& Silvers WK (1985). Distribution of ATPase-positive Langerhans cells in normal adult human skin. British Journal of Dermatology, 113: 707-711. 